\title{
Vertex Constraints in 3D Higher Spin Theories
}

\author{
Stefan Fredenhagen ${ }^{*} *$ \\ University of Vienna, Faculty of Physics, Boltzmanngasse 5, 1090 Vienna, Austria \\ and Erwin Schrödinger International Institute for Mathematics and Physics, Boltzmanngasse 9, 1090 Vienna, Austria \\ Olaf Krüger ${ }^{\dagger}$ \\ University of Vienna, Faculty of Physics, Boltzmanngasse 5, 1090 Vienna, Austria \\ Karapet Mkrtchyan $\oplus^{\ddagger}$ \\ Max Planck Institute for Gravitational Physics (Albert Einstein Institute), Am Mühlenberg 1, 14476 Potsdam, Germany
}

(Received 28 June 2019; published 25 September 2019)

\begin{abstract}
We analyze the constraints imposed by gauge invariance on higher-order interactions between massless bosonic fields in three-dimensional higher-spin gravities. Focusing on the transverse-traceless part, we show that vertices of quartic and higher order that are independent of the cubic ones can only involve scalars and Maxwell fields. As a consequence, the full nonlinear interactions of massless higher-spin fields are completely fixed by the cubic vertex.
\end{abstract}

DOI: $10.1103 /$ PhysRevLett.123.131601

Introduction.-In this Letter, we start an investigation aimed at a Lagrangian formulation of three-dimensional (3D) higher-spin (HS) gravities [1] beyond cubic order.

HS gravity theories are generalizations of gravity, where higher-spin gauge fields are introduced. In $3 \mathrm{D}$, a free spin- $s$ gauge field is a symmetric tensor field $\phi_{\mu_{1} \cdots \mu_{s}}$ with gauge transformation

$$
\delta^{(0)} \phi_{\mu_{1} \cdots \mu_{s}}=s \partial_{\left(\mu_{1}\right.} \epsilon_{\left.\mu_{2} \cdots \mu_{s}\right)},
$$

similar to Maxwell or Chern-Simons vector gauge fields $(s=1)$ and linearized gravity $(s=2)$. It is described by the quadratic Fronsdal Lagrangian $\mathcal{L}_{2}$ [2]. We collectively denote massless fields with spin $s>1$ and ChernSimons vector fields as "massless HS fields." In 3D, these do not possess propagating degrees of freedom; however, they can have interesting boundary dynamics at the conformal boundary of asymptotically anti-de Sitter (AdS) space-times. Up to now, no nonlinear Lagrangian of interacting Fronsdal fields is known, but there is a systematic perturbative approach to construct such Langrangians. This is known as the Noether-Fronsdal program, which we follow in this work and review below.

There are different motivations to study HS gravities. Most prominently, they constitute generalizations of gravity for which holographic dualities can be investigated: a

Published by the American Physical Society under the terms of the Creative Commons Attribution 4.0 International license. Further distribution of this work must maintain attribution to the author(s) and the published article's title, journal citation, and DOI. Funded by SCOAP ${ }^{3}$. $(d+1)$-dimensional HS gravity theory on asymptotically AdS space-times is related to a $d$-dimensional conformal field theory (CFT). This HS AdS/CFT correspondence $[3,4]$ is a priori independent of the string-theoretic AdS/CFT correspondence, and possesses distinct features as it does not require supersymmetry and is accessible to perturbative checks. It becomes particularly interesting for 3D HS gravities [5], because for 2D CFTs many exact results are available. These also allow one to study the relation between the tensionless limit of string theory and HS theories via their CFT dual $[6,7]$.

To perform computations on the HS side, finding a Lagrangian formulation is crucial. For the nonpropagating sector (i.e., without scalars or Maxwell fields), a nonlinear action is available in Chern-Simons form [8-10] (which is a generalization of the Chern-Simons formulation of 3D gravity [11]). There, one uses the framelike formulation of HS fields in terms of generalized vielbein fields and spin connections. In this formulation, coupling to matter is not straightforward. It can be achieved by following the Vasiliev approach [12] which uses infinitely many auxiliary fields and for which no standard action is known.

The metriclike formulation of HS gravity [13-17] (based on Fronsdal fields) is more suitable for matter coupling. For example, the cubic interactions of massless HS fields are well studied both in flat [18-28] and (A)dS spaces [29-34] of dimensions $D \geq 4$. However, the main challenge in formulating the action in arbitrary dimensions arises at quartic order (see, e.g., Refs. [35-42]) and this is also expected in the 3D case with matter.

In the Noether procedure one starts with the free quadratic Lagrangian $\mathcal{L}_{2}$ and builds vertices order by order, 
including matter couplings. For a given HS theory, we expand the Lagrangian in powers of small parameters $g_{n}$,

$$
\mathcal{L}=\mathcal{L}_{2}+\sum_{n \geq 3} g_{n} \mathcal{L}_{n}+O\left(g_{n}^{2}\right)
$$

where we suppress a sum over the different kinds of $n$-point vertices $\mathcal{L}_{n}$. Altogether, $\mathcal{L}$ must be gauge invariant, $\delta \mathcal{L}=0$, up to boundary terms, where $\delta$ is obtained by deforming the transformation of the free fields [see Eq. (1)],

$$
\delta=\delta^{(0)}+\delta^{(1)}+\cdots,
$$

expanded in powers of the fields.

Cubic gauge invariant vertices in 3D have been classified $[16,17]$. In this work, we study higher-order vertices of massless fields that are independent of the ones of lower order. Because of gauge invariance, they satisfy the following Noether equations:

$$
\delta^{(n-2)} \mathcal{L}_{2}+\delta^{(0)} \mathcal{L}_{n}=0 \quad \text { up to total derivatives. }
$$

Assuming that nontrivial vertices are nonzero when restricted to the transverse-traceless sector, we show that after suitable field redefinitions in 3D there are no independent vertices of order $n \geq 4$ that contain massless higher-spin fields.

Preliminaries.-The Lagrangian $\mathcal{L}$ is written in terms of massless Fronsdal fields, subject to nonlinear gauge transformations. For the classification, we focus on the traceless and transverse (TT) part of the vertices, i.e., the part that does not contain divergences and traces of the fields. We briefly comment on the relation to the full vertices in the conclusion. Hence, from now on we assume that the fields are parametrized by symmetric, traceless, and divergencefree tensors $\phi_{\mu_{1} \cdots \mu_{s}}(x)$ with $\mu_{i} \in(0,1,2) ; s$ denotes the spin of the field and the corresponding free equation of motion $(\mathrm{EOM})$ is the Klein-Gordon equation with zero mass (see, e.g., Ref. [17]).

For convenience, one contracts the tensor indices each with an auxiliary vector variable $a^{\mu}$. This defines

$$
\phi^{(s)}(x, a)=\frac{1}{s !} \phi_{\mu_{1} \cdots \mu_{s}}(x) a^{\mu_{1}} \cdots a^{\mu_{s}}
$$

and the properties of $\phi_{\mu_{1} \cdots \mu_{s}}(x)$ translate to the Fierz equations for $\phi^{(s)}(x, a)$ :

$$
A^{2} \phi^{(s)}=A \cdot P \phi^{(s)}=\left.P^{2} \phi^{(s)}\right|_{\text {free EOM }}=0,
$$

where $P^{\mu}=\partial_{x^{\mu}}$ and $A^{\mu}=\partial_{a^{\mu}}$.

We analyze the general form of the deformations $\mathcal{L}_{n}$ for $n \geq 4$, which can be written as

$$
\mathcal{L}_{n}=\left.\mathcal{V}\left(\prod_{i=1}^{n} \phi_{i}\left(x_{i}, a_{i}\right)\right)\right|_{\substack{x_{i}=x \\ a_{i}=0}},
$$

where we abbreviated $\phi_{i}=\phi^{\left(s_{i}\right)}$. The vertex generating operator $\mathcal{V}$ performs the index contractions via the operators $P_{i}^{\mu}=\partial_{x_{i}^{\mu}}$ and $A_{i}^{\mu}=\partial_{a_{i}^{\mu}}$. Let us first concentrate on parity even vertices $\mathcal{L}_{n}$; hence $\mathcal{V}$ is a polynomial in the commuting variables

$$
z_{i j}=A_{i} \cdot A_{j}, \quad y_{i j}=A_{i} \cdot P_{j}, \quad s_{i j}=P_{i} \cdot P_{j} .
$$

These contract two indices each: One from $\phi_{i}$ with one from $\phi_{j}\left(z_{i j}\right)$; one from $\phi_{i}$ with one from a derivative acting on $\phi_{j}\left(y_{i j}\right)$; and two from derivatives acting on $\phi_{i}$ and $\phi_{j}$ $\left(s_{i j}\right)$. The $s_{i j}$ are the familiar Mandelstam variables. In the end, we set $a_{i}=0$ to ensure Lorentz invariance. Whenever appropriate, we use an $n$-periodic index notation, e.g., $s_{i n+j}=s_{i j}$.

Equivalence relations.-We say that two vertex generating operators $\mathcal{V}$ and $\mathcal{V}^{\prime}$ are equivalent, $\mathcal{V} \approx \mathcal{V}^{\prime}$, if and only if the two resulting Lagrangians $\mathcal{L}_{n}$ and $\mathcal{L}_{n}^{\prime}$, constructed via Eq. (4), describe the same theory. Hence, we seek the most general form of $\mathcal{V}$, up to equivalence.

For example, field redefinitions $\phi_{i} \mapsto \phi_{i}+\delta \phi_{i}$, such that $\delta \phi_{i}$ is of order $n-1$ in the fields, do not alter the theory, but change $\mathcal{L}_{n}$ by terms proportional to the free equation of motion of $\phi_{i}$ whose TT part is just the massless wave equation. Choosing $\delta \phi_{i}$ properly, we can remove all appearances of $s_{i i}=P_{i}^{2}$ in the TT part of $\mathcal{L}_{n}$ for any $n$ and all $i=1, \ldots, n$. This generalizes the so-called Metsaev basis for cubic vertices $[17,20,21,28]$ to higher $n$.

Since we are interested in the TT part of the vertex, $\mathcal{V}$ does not depend on $z_{i i}$ and $y_{i i}$. So far, we summarize that $\mathcal{V}$ is an element in the polynomial ring $\mathcal{R}=\mathbb{R}\left[\left.z_{i j}\right|_{i<j},\left.y_{i j}\right|_{i \neq j},\left.s_{i j}\right|_{i<j}\right]$.

Furthermore, acting with $D^{\mu}=\sum_{j=1}^{n} P_{j}^{\mu}$ on the expression in brackets in Eq. (4) results in a total derivative term in the Lagrangian which does not affect the theory. We may hence remove any dependence of $\mathcal{V}$ on $A_{i} \cdot D$ and $P_{i} \cdot D$. In other words, we impose the equivalence relations

$$
\sum_{j=1}^{n} y_{i j} \approx 0, \quad \sum_{j=1}^{n} s_{i j} \approx 0
$$

which generate an ideal $\mathcal{I}_{D} \subset \mathcal{R}$.

A final class of equivalence relations is given by Schouten identities, which stem from over-antisymmetrization of space-time indices within the Lagrangian. They translate to an ideal $\mathcal{I}_{S}$ of equivalence relations in $\mathcal{R}$ as follows: Consider the vector of derivative operators $b=$ $\left(P_{1}, \ldots, P_{n}, A_{1}, \ldots, A_{n}\right)$ and the symmetric $2 n \times 2 n$ matrix 


$$
\mathcal{B}=\left.\left(b_{K} \cdot b_{L}\right)\right|_{K, L \in(1, \ldots, 2 n)}=\left(\begin{array}{cc}
\mathcal{S} & \mathcal{Y}^{T} \\
\mathcal{Y} & \mathcal{Z}
\end{array}\right) .
$$

Here, $\mathcal{S}=\left(s_{i j}\right), \mathcal{Y}=\left(y_{i j}\right), \mathcal{Z}=\left(z_{i j}\right)$ are $n \times n$ matrices with elements in $\mathcal{R}$ (hence, their diagonal elements vanish). Now, remove $2 n-4$ rows and columns from $\mathcal{B}$ and call the resulting $4 \times 4$ matrix $M$. Acting with $\operatorname{det} M$ on the term in brackets in Eq. (4) yields an expression with four antisymmetrized space-time indices, which vanishes in three dimensions. All such $4 \times 4$ minors of $\mathcal{B}$ form a generating set for the ideal $\mathcal{I}_{S}$.

All in all, $\mathcal{V}$ is a representative of an equivalence class in the quotient ring $[\mathcal{V}] \in \mathcal{R} /\left(\mathcal{I}_{D}+\mathcal{I}_{S}\right)$ and we are free to choose a convenient one, since all generating operators in one equivalence class describe the same vertex. However, it is hard to find simple representatives, because the ideal $\mathcal{I}_{S}$ is too complicated. In the next section, we show that it is easier to get a hold on representatives of $[\Delta \mathcal{V}]$, where we multiply $\mathcal{V}$ by an appropriate product $\Delta$ of Mandelstam variables $s_{i j}$. The operator $\Delta \mathcal{V}$ corresponds to acting with contracted space-time derivatives on the vertex generated by $\mathcal{V}$. Then, by choosing a simple representative for $[\Delta \mathcal{V}]$, we can impose strong constraints on the vertex generating operator $\mathcal{V}$ itself. We show this shortly.

$\mathcal{V}$ and $\Delta \mathcal{V}$ generate physically distinct vertices. However, if one of them is trivial, so is the other. In particular, as we show in the rest of this section,

$$
\Delta \mathcal{V} \approx 0 \Rightarrow \mathcal{V} \approx 0
$$

This can be seen in Fourier space, where the operators $s_{i j}$ can be treated as numbers. If $\Delta$ is a product of $s_{i j}(i \neq j)$, then it is generically nonzero on the subvariety in $k$ space defined by $k_{i}^{2}=0$ and $\sum k_{i}=0$. The property $\Delta \mathcal{V} \approx 0$ translates in Fourier space to the condition that $\Delta \mathcal{V}$ applied on any product of fields $\hat{\phi}_{i}\left(k_{i}, a_{i}\right)$ (evaluated at $a_{i}=0$ ) vanishes on this subvariety. As $\Delta$ is nonvanishing almost everywhere and $\mathcal{V}$ only depends polynomially on $k_{i}^{\mu}$, one concludes that $\mathcal{V}$ applied on the fields $\hat{\phi}_{i}$ vanishes, hence $\mathcal{V} \approx 0$.

Choice of representatives. - In this section, we use Schouten identities to choose a convenient representative of $[\Delta \mathcal{V}]$ for a given vertex generating operator $\mathcal{V}$ multiplied by a suitable product $\Delta$ of Mandelstam variables $s_{i j}$. First, let $M$ be a $4 \times 4$ submatrix of $\mathcal{B}$ containing the first three rows and columns, as well as the $(n+i)$ th row and $(n+j)$ th column with $i \neq j$. Using the corresponding Schouten identity

$$
0 \approx \operatorname{det} M=2 s_{12} s_{23} s_{31} z_{i j}+\left(\text { terms independent of } z_{k l}\right),
$$

we can replace $z_{i j}$ in $\Delta \mathcal{V}$ by the $y_{k l}$ and $s_{k l}$ variables if $\Delta$ is chosen to contain high enough powers of $s_{12} s_{23} s_{31}$. Doing this for all pairs $(i \neq j)$ allows us to choose a representative for $[\Delta \mathcal{V}]$ that does not depend on any $z_{i j}$. In a similar fashion we can reduce the number of $y_{k l}$ variables: Pick out a $4 \times 4$ submatrix $M$ of $\mathcal{B}$ including the columns $i, i+1$, $i+2$ (modulo $n$ ) and $(n+i)$, such that the latter contains the elements $y_{i i}=0, y_{i i+1}, y_{i i+2}$ and any other, say $y_{i j}$. Then the Schouten identities $\operatorname{det} M \approx 0$ can be used to replace all of the operators $y_{i j}$ in $\Delta \mathcal{V}$ by $y_{i i+1}, y_{i i+2}$ and the Mandelstam variables. Finally, we perform a change of variables by replacing each $y_{i i+2}$ in $\Delta \mathcal{V}$ by a linear combination of $y_{i i+1}$ and $Y_{i}:=s_{i i+2} y_{i i+1}-s_{i i+1} y_{i i+2}$. The reason for this replacement becomes more apparent in the next section, but note for now that $Y_{i}^{2} \approx 0$ due to Schouten identities. Indeed, the $4 \times 4 \operatorname{minor} \operatorname{det} M$ of $\mathcal{B}$, which consists of the rows and columns $i, i+1, i+2$ (modulo $n$ ), and $i+n$, satisfies $\operatorname{det} M=Y_{i}^{2}$.

We conclude that for a given vertex generating operator $\mathcal{V}$, there exists a product of Mandelstam variables $\Delta$, such that

$$
\Delta \mathcal{V} \approx Q_{\mathcal{V}}\left(y_{i i+1}, Y_{i}, s_{i j}\right),
$$

and the polynomial $Q_{\mathcal{V}}$ is at most linear in each $Y_{i}$ (a term $Y_{i} Y_{j}$ with $i \neq j$ is still possible, but $Y_{i}^{2}$ is not). We note that the polynomial might not be unique. It can be seen as a representative of an equivalence class

$$
\left[Q_{\mathcal{V}}\right] \in \frac{\mathbb{R}\left[y_{i i+1}, Y_{i}, s_{i j}\right]}{\mathcal{I}_{R}+\left\langle Y_{i}^{2}\right\rangle},
$$

where the ideal $\mathcal{I}_{R} \subset \mathbb{R}\left[y_{i i+1}, Y_{i}, s_{i j}\right]$ is generated by all remaining equivalence relations (total derivatives and Schouten identities).

Constraints from gauge invariance.-In this section, we show that gauge invariance implies that the polynomial $Q_{\mathcal{V}}$ in Eq. (6) does not depend on $y_{i i+1}$. To this end, we consider the 0th order gauge transformations of the fields [see Eq. (1)],

$$
\delta^{(0)} \phi^{(s)}(x, a)=a \cdot P \epsilon^{(s-1)}(x, a),
$$

where the gauge parameter $\epsilon^{(s-1)}$, constructed as in Eq. (3), also satisfies the Fierz equations.

In the condition for gauge invariance, Eq. (2), the first term vanishes when the free equations of motion are applied. Hence,

$$
\delta_{k}^{(0)} \mathcal{L}_{n}=\left.\mathcal{V} a_{k} \cdot P_{k}\left(\epsilon_{k}\left(x_{k}, a_{k}\right) \prod_{1 \leq i \leq n}^{i \neq k} \phi_{i}\left(x_{i}, a_{i}\right)\right)\right|_{\substack{x_{i}=x \\ a_{i}=0}}
$$

must vanish up to total derivatives when the Fierz equations for $\phi_{i}$ and $\epsilon_{k}$ are imposed. We deduce that the corresponding vertex generating operator $\mathcal{V} \in \mathcal{R}$ satisfies $\left[\mathcal{V}, a_{k} \cdot P_{k}\right] \approx 0$ for $k=1, \ldots, n$. The operators $a_{k} \cdot P_{k}$ commute with $s_{i j}$, hence, $\left[\Delta \mathcal{V}, a_{k} \cdot P_{k}\right] \approx 0$ for any product $\Delta$ of Mandelstam 
variables, and since the ideal $\mathcal{I}_{S}+\mathcal{I}_{D}$ is gauge invariant, we find that

$$
\left[Q_{\mathcal{V}}\left(y_{i i+1}, Y_{i}, s_{i j}\right), a_{k} \cdot P_{k}\right] \approx 0 .
$$

Using

$$
\left[y_{i i+1}, a_{k} \cdot P_{k}\right]=\delta_{i k} s_{i i+1}, \quad\left[Y_{i}, a_{k} \cdot P_{k}\right]=0,
$$

this reduces to

$$
s_{k k+1} \partial_{y_{k k+1}} Q_{\mathcal{V}}\left(y_{i i+1}, Y_{i}, s_{i j}\right) \approx 0,
$$

where $Y_{i}$ is now treated as an independent variable: $\partial_{y_{k k+1}} Y_{i}=0$.

Note that all remaining equivalence relations in $\mathcal{I}_{R}$ are gauge invariant; hence, the generators of $\mathcal{I}_{R}$ can be chosen to be polynomials only in $Y_{i}$ and $s_{i j}$. We conclude that because of Eq. (7), $Q_{\mathcal{V}}$ can be chosen to be independent of $y_{i i+1}$.

Parity-odd vertices.-So far, we have only discussed parity-even deformations. The most general form of a parity-odd $n$-point vertex $\mathcal{L}_{n}$ is also given by Eq. (4), but with the vertex generating operator $\mathcal{V}$ replaced by a linear combination $\mathcal{W}$ of operators $\mathcal{V} B_{I J K}$, where $\mathcal{V} \in \mathcal{R}$ and

$$
B_{I J K}=\epsilon_{\mu \nu \rho} b_{I}^{\mu} b_{J}^{\nu} b_{K}^{\rho}, \quad I, J, K=1, \ldots, 2 n
$$

contains a single epsilon tensor. Let $s_{3}$ be the $3 \times 3$ matrix that consists of the first three rows and columns of $\mathcal{S}$. Then, det $s_{3}=2 s_{12} s_{13} s_{23}$ is a product of Mandelstam variables and

$$
\operatorname{det} s_{3} \cdot B_{I J K}=\frac{1}{6}\left(\mathcal{B}_{1 I} \mathcal{B}_{2 J} \mathcal{B}_{3 K} \pm 5 \text { terms }\right) \cdot B_{123} .
$$

This relation is proved using det $s_{3}=\left(B_{123}\right)^{2}$.

We can now conclude along the lines of the previous sections: For a given parity-odd $n$-point vertex $\mathcal{L}_{n}$, there exists a product $\Delta$ of Mandelstam variables, such that the corresponding vertex generating operator $\mathcal{W}$ satisfies

$$
\Delta \mathcal{W} \approx Q_{\mathcal{W}}\left(Y_{i}, s_{i j}\right) \cdot B_{123},
$$

where the polynomial $Q_{\mathcal{W}}$ is linear in the $Y_{i}$ 's. The only additional input along this proof is that $B_{123}$ is gauge invariant $\left(\left[B_{123}, a_{k} \cdot P_{k}\right]=0\right)$.

Final steps.-Let us summarize: A Lorentz and gauge invariant parity-even $n$-point vertex $\mathcal{L}_{n}$ is given by Eq. (4) and there exists a product $\Delta$ of Mandelstam variables, such that the vertex generating operator $\Delta \mathcal{V}$ is equivalent to a polynomial $Q_{\mathcal{V}}\left(Y_{i}, s_{i j}\right)$, which is linear in each $Y_{i}$. This means that there is no product $A_{i}^{\mu} A_{i}^{\nu}$ left, when we write $Q_{\mathcal{V}}$ in terms of the operators $P_{i}^{\mu}$ and $A_{i}^{\mu}$. The equivalence relations do not change the number of those operators, so this must also be true for $\Delta \mathcal{V}$. Finally, $s_{i j}$ (and thus, $\Delta$ ) only consist of the operators $P_{i}^{\mu}$. We conclude that $\mathcal{V}$ cannot contain any product $A_{i}^{\mu} A_{i}^{\nu}$, meaning that the corresponding $n$-point vertex $\mathcal{L}_{n}$, constructed via Eq. (4), may only involve fields whose spin is at most one. Note that for this argument, Eq. (5) is essential.

For a parity-odd vertex, we use an analogous reasoning, except that $\Delta \mathcal{W}$ is equivalent to a polynomial $Q_{\mathcal{W}}\left(Y_{i}, s_{i j}\right)$ multiplied with $Q_{123}$. But since $Q_{123}$ does not contain any $A_{i}^{\mu}$ operator, this does not alter the conclusion.

Finally, we find the extra equivalence relations $Y_{i} \approx 0$ for Chern-Simons fields $\phi_{i}$, which stem from the corresponding free EOM. Hence, $\mathcal{L}_{n}$ may only contain massless scalars and Maxwell fields, i.e., fields with propagating degrees of freedom. This completes the proof of the statement in the Introduction: there are no independent vertices of order $n \geq 4$ that contain massless HS fields.

Conclusions. - We have shown in this Letter that in three dimensions gauge invariance strongly constrains the higher-order interactions that involve massless fields. In particular, vertices that are independent of the cubic ones can only contain scalars and Maxwell fields, but no massless HS fields. Our argument even applies when we extend the set of propagating fields by massive bosons (scalar, Proca, or higher-spin massive fields): Gauge invariance is so strong that it forbids massless HS fields from entering any independent higher-order vertex irrespective of the remaining field content of the theory. The proof goes along the same way, except that for $\Delta$ in Eq. (5) we have to consider a more general nonzero polynomial (instead of a monomial) in the Mandelstam variables. We will elaborate on this case in Ref. [43]. Furthermore, although the results were derived in flat space-time, they also hold for (A)dS (or even any Einstein background) due to an argument given for the cubic vertices in Ref. [16].

In the classification we concentrated on the TT part of the vertices. By our analysis it is not excluded that there are higher order vertices with vanishing TT part. However, there are several reasons to expect that the TT part contains the relevant physical information about interactions: First, most of the individual terms containing a trace and/or a divergence can be set to zero by a gauge choice (even all of them when the fields are on shell), so it seems that such terms do not carry gauge-invariant information. Second, in the Fronsdal formulation no case of a vertex with a vanishing TT part is known. In a different formulation such a possibility is known (see Section IV. 5 of Ref. [34]), but also there it was observed that the corresponding vertex does not affect physics. Therefore, we ignore potential subtleties related to the non-TT part.

Our result implies that in any nonlinear theory with a HS spectrum, all higher-order vertices that only include massless HS fields arise by the completion of the cubic ones to the full nonlinear Lagrangian (as in Yang-Mills theory or 
general relativity). This has an interesting consequence in holography. In Refs. [16,17] it was observed that cubic vertices satisfy triangle inequalities for the spins. Our result implies that the only higher-order vertices are the completions of the cubic ones, and they can be shown to satisfy polygon inequalities. This is in agreement with the CFT prediction [44] and establishes a one-to-one map between bulk vertices and boundary correlators in the context of $\mathrm{AdS}_{3} / \mathrm{CFT}_{2}$.

HS fields are known to admit a Chern-Simons description in three dimensions, in a first-order formulation. The findings of this Letter imply that all the fields that do not carry bulk propagating degrees of freedom, cannot participate in independent higher-order interactions. This is consistent with the statement on the absence of higherorder self-interactions of Chern-Simons fields [45] and may indicate an exact equivalence of the Chern-Simons and metric descriptions of HS fields in the gauge sector. It is therefore tempting to speculate that any nonlinear action of massless HS fields without matter can be written in a Chern-Simons form. Such an equivalence cannot extend to the matter sector though.

It would be interesting to extend this work to higher dimensions. In that case there will be independent higherorder interactions for HS gauge fields. The classification of the vertices satisfying Eq. (2) in arbitrary dimensions will be given in Ref. [43].

The authors are grateful to Andrea Campoleoni, Dario Francia, Maxim Grigoriev, Euihun Joung, Thorsten Schimannek, and Arkady Tseytlin for useful discussions. The hospitality of the Erwin Schrödinger International Institute for Mathematics and Physics during the program on "Higher Spins and Holography" is greatly appreciated.

*stefan.fredenhagen@univie.ac.at

†olaf.krueger@univie.ac.at

*karapet.mkrtchyan@aei.mpg.de

[1] S. F. Prokushkin and M. A. Vasiliev, Nucl. Phys. B545, 385 (1999).

[2] C. Fronsdal, Phys. Rev. D 18, 3624 (1978).

[3] I. R. Klebanov and A. M. Polyakov, Phys. Lett. B 550, 213 (2002).

[4] S. Giombi and X. Yin, J. Phys. A 46, 214003 (2013).

[5] M. R. Gaberdiel and R. Gopakumar, Phys. Rev. D 83, 066007 (2011).

[6] M. R. Gaberdiel and R. Gopakumar, J. High Energy Phys. 11 (2014) 044.

[7] M. R. Gaberdiel and R. Gopakumar, J. High Energy Phys. 09 (2016) 085.

[8] M. P. Blencowe, Classical Quantum Gravity 6, 443 (1989).

[9] A. Campoleoni, S. Fredenhagen, S. Pfenninger, and S. Theisen, J. High Energy Phys. 11 (2010) 007.

[10] H. Afshar, A. Bagchi, R. Fareghbal, D. Grumiller, and J. Rosseel, Phys. Rev. Lett. 111, 121603 (2013).
[11] A. Achucarro and P. K. Townsend, Phys. Lett. B 180, 89 (1986).

[12] S. Prokushkin and M. A. Vasiliev, arXiv:hep-th/9812242.

[13] A. Campoleoni, S. Fredenhagen, S. Pfenninger, and S. Theisen, J. Phys. A 46, 214017 (2013).

[14] S. Fredenhagen and P. Kessel, J. Phys. A 48, 035402 (2015).

[15] A. Campoleoni and M. Henneaux, J. High Energy Phys. 03 (2015) 143.

[16] K. Mkrtchyan, Phys. Rev. Lett. 120, 221601 (2018).

[17] P. Kessel and K. Mkrtchyan, Phys. Rev. D 97, 106021 (2018).

[18] A. K. H. Bengtsson, I. Bengtsson, and N. Linden, Classical Quantum Gravity 4, 1333 (1987).

[19] R. R. Metsaev, Nucl. Phys. B759, 147 (2006).

[20] R. Manvelyan, K. Mkrtchyan, and W. Rühl, Nucl. Phys. B844, 348 (2011).

[21] R. Manvelyan, K. Mkrtchyan, and W. Rühl, Nucl. Phys. B836, 204 (2010).

[22] A. Sagnotti and M. Taronna, Nucl. Phys. B842, 299 (2011).

[23] A. Fotopoulos and M. Tsulaia, J. High Energy Phys. 11 (2010) 086.

[24] R. Manvelyan, K. Mkrtchyan, and W. Rühl, Phys. Lett. B 696, 410 (2011).

[25] K. Mkrtchyan, Phys. At. Nucl. 75, 1264 (2012).

[26] R. R. Metsaev, Phys. Lett. B 720, 237 (2013).

[27] M. Henneaux, G. Lucena-Gómez, and R. Rahman, J. High Energy Phys. 01 (2014) 087.

[28] E. Conde, E. Joung, and K. Mkrtchyan, J. High Energy Phys. 08 (2016) 040.

[29] E. S. Fradkin and M. A. Vasiliev, Nucl. Phys. B291, 141 (1987).

[30] M. A. Vasiliev, Nucl. Phys. B862, 341 (2012).

[31] E. Joung and M. Taronna, Nucl. Phys. B861, 145 (2012).

[32] R. Manvelyan, R. Mkrtchyan, and W. Rühl, Nucl. Phys. B872, 265 (2013).

[33] N. Boulanger, D. Ponomarev, and E. D. Skvortsov, J. High Energy Phys. 05 (2013) 008.

[34] D. Francia, G. L. Monaco, and K. Mkrtchyan, J. High Energy Phys. 04 (2017) 068.

[35] F. A. Berends, G. J. H. Burgers, and H. van Dam, Nucl. Phys. B260, 295 (1985).

[36] M. Taronna, J. High Energy Phys. 04 (2012) 029.

[37] X. Bekaert, J. Erdmenger, D. Ponomarev, and C. Sleight, J. High Energy Phys. 11 (2015) 149.

[38] A. K. H. Bengtsson, J. High Energy Phys. 12 (2016) 134.

[39] M. Taronna, J. High Energy Phys. 05 (2017) 026.

[40] R. Roiban and A. A. Tseytlin, J. High Energy Phys. 04 (2017) 139.

[41] C. Sleight and M. Taronna, Phys. Rev. Lett. 121, 171604 (2018).

[42] D. Ponomarev, Universe 4, 2 (2018).

[43] S. Fredenhagen, O. Krüger, and K. Mkrtchyan (to be published).

[44] S. Fredenhagen, O. Krüger, and K. Mkrtchyan, arXiv: 1812.10462.

[45] G. Barnich and M. Henneaux, Phys. Lett. B 311, 123 (1993). 